\title{
Perspective review on solid-organ transplant: needs in point-of-care optical biomarkers
}

\author{
Daqing Piao \\ Alan Hawxby \\ Harlan Wright \\ Erin M. Rubin
}




\title{
Perspective review on solid-organ transplant: needs in point-of-care optical biomarkers
}

\author{
Daqing Piao, a,b,* Alan Hawxby, ${ }^{\mathrm{c}}$ Harlan Wright, ${ }^{\mathrm{c}}$ and Erin M. Rubin ${ }^{\mathrm{d}}$ \\ a Oklahoma State University, School of Electrical and Computer Engineering, Stillwater, Oklahoma, United States \\ ${ }^{b}$ Oklahoma State University, Department of Veterinary Clinical Sciences, Center for Veterinary Health Sciences, Stillwater, Oklahoma, \\ United States \\ 'University of Oklahoma Health Sciences Center, Oklahoma Transplant Center, Oklahoma City, Oklahoma, United States \\ 'University of Oklahoma Health Sciences Center, Department of Pathology, Oklahoma City, Oklahoma, United States
}

\begin{abstract}
Solid-organ transplant is one of the most complex areas of modern medicine involving surgery. There are challenging opportunities in solid-organ transplant, specifically regarding the deficiencies in pathology workflow or gaps in pathology support, which may await alleviations or even de novo solutions, by means of point-of-care, or point-of-procedure optical biomarkers. Focusing the discussions of pathology workflow on donor liver assessment, we analyze the undermet need for intraoperative, real-time, and nondestructive assessment of the donor injuries (such as fibrosis, steatosis, and necrosis) that are the most significant predictors of post-transplant viability. We also identify an unmet need for real-time and nondestructive characterization of ischemia or irreversible injuries to the donor liver, earlier than appearing on morphological histology examined with light microscopy. Point-of-procedure laparoscopic optical biomarkers of liver injuries and tissue ischemia may also facilitate post-transplant management that is currently difficult for or devoid of pathological consultation due to lack of tools. The potential and pitfalls of point-of-procedure optical biomarkers for liver assessment are exemplified in breadth for steatosis. The more general and overarching challenges of point-of-procedure optical biomarkers for liver transplant pathology, including the shielding effect of the liver capsule that was quantitated only recently, are projected. The technological and presentational benchmarks that a candidate technology of point-of-procedure optical biomarkers for transplant pathology must demonstrate to motivate clinical translation are also foreseen. (c) 2018 Society of Photo-Optical Instrumentation Engineers (SPIE) [DOI: 10.1117/1.JBO.23.8.080601]
\end{abstract}

Keywords: solid-organ transplant; optical biomarker; point-of-care; fibrosis; steatosis; optical spectroscopy.

Paper 180376-PER received Jun. 23, 2018; accepted for publication Aug. 2, 2018; published online Aug. $29,2018$.

\section{Introduction}

Being one of the most complex areas of medical discipline involving surgical procedures over an open operative field that has well-defined anatomy and no inconspicuous tissue margin, solid-organ transplant ${ }^{1-5}$ seems to invite no ground for diagnostic or therapeutic applications of the light-based technologies that are cradled as biomedical optics and have thrived in applications toward cancer diagnosis ${ }^{6}$ and surgery planning. ${ }^{7}$ However, there are concerns affecting patient-management in solid-organ transplant that may await alleviation by means of biomedical optics technologies. There are also deficiencies impacting patient management in solid-organ transplant that call for de novo solutions by means of biomedical optics innovations. The concerns and deficiencies in solid-organ transplant poised for injecting technologies, including those of biomedical optics, may reside specifically in where the pathology support or pathology-surrogating information could be more readily available than it is now.

Concerns regarding pathology support that impact clinical management of solid-organ transplant are exemplified, in our perspectives, in the pathology workflow for liver transplant. In this article, we outline the current standard workflow of pathology support in liver transplant according to the technological

*Address all correspondence to: Daqing Piao, E-mail: daqing.piao@okstate .edu aspects impacting the robustness of the generated clinical information while the pathology evaluation is a routine or when a pathology consultation is called upon. The viewpoints on the technological aspects potentiate a workpoint of applying biomedical-optics technologies to donor assessment for liver transplant in the capacity of biomarkers ${ }^{8,9}$ interrogated optically as the point-of-care, or point-of-procedure, optical biomarkers. Point-of-procedure optical biomarkers refer to light-based diagnoses for real-time, nondestructive, and whole organ assessment of donor organ injuries that are predictive of post-transplant outcomes. Information of the donor organ impacting posttransplant outcomes may include morphologically manifested injuries such as steatosis and fibrosis, which are identifiable by routine pathology and functionally enacted injuries, such as acute ischemia that could be underdetected or undetected by routine light microscopy-based histopathology. In discussing undermet and unmet needs in the pathology workflow specific to pretransplant donor liver assessment, we project potential and pitfalls of optical biomarkers toward point-of-procedure assessment of donor organ injuries that are likely the most predictive of post-transplant outcomes and identification of ischemia. The potential of optical biomarkers for real-time, nondestructive, and whole organ assessment, as well as ischemia identification, may also render point-of-procedure assessment in areas that are currently difficult for or devoid of pathological consultation due to lack of tools. We expect that the de novo applications of point-of-procedure optical biomarkers could be useful for 


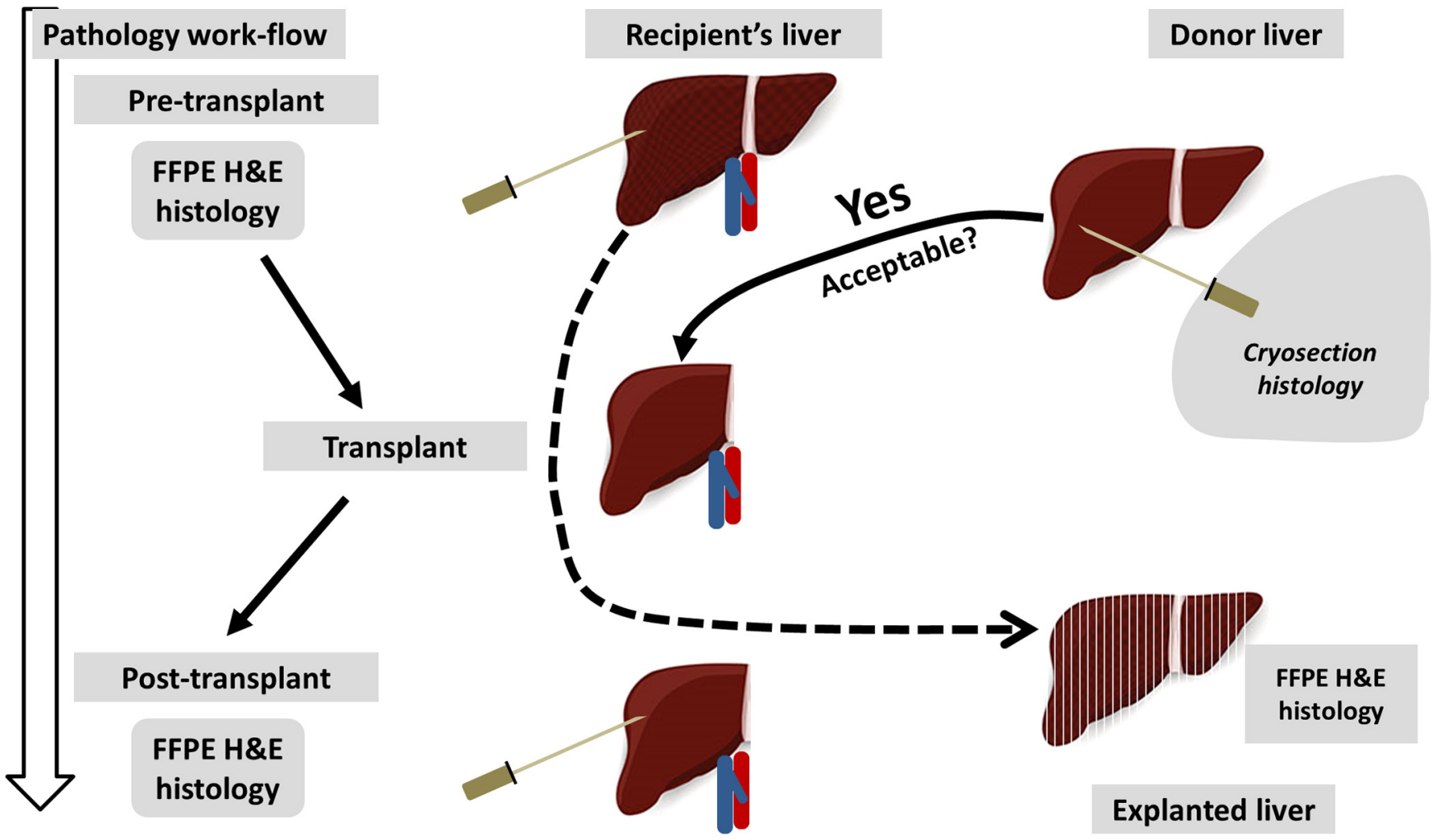

Fig. 1 Pathology workflow for liver transplant. Pretransplant pathology assesses the liver of the recipient for transplant candidacy and the donor liver for transplant suitability. Paratransplant pathology diagnoses the liver explanted from the recipient to primarily confirm the pretransplant assessment. Post-transplant pathology examines the allograft liver to support the clinical management of allograft survival and long-term functioning.

laparoscopic assessment of the recipient's liver in pretransplant workout and minimally invasive identification of the ischemic site, as well as for assessment of allograft health during posttransplant management.

\section{Pathology Workflow in Liver Transplant}

Liver transplant is unquestionably a convoluted frontier for histopathologists whose opinions are crucial to all phases of patient management. ${ }^{10}$ In liver transplant, a histopathologist contributes to the decision-making process from pre- to post-transplant settings, in multiple stages of patient management and at multiple time points, as conceptualized in Fig. 1. The roles of a histopathologist in liver transplant are stationed over three phases: (1) pretransplant diagnosis of the recipient's liver disease and assessment of the donor liver suitability; (2) paratransplant examination of the explanted liver; and (3) post-transplant evaluation of the allograft viability.

\subsection{Pretransplant Pathology in Liver Transplant: Diagnosis of the Recipient's Liver}

Liver transplant is considered a highly successful treatment ${ }^{11}$ to liver failure caused by end-stage liver disease (ESLD), fulminant hepatic failure, and early-stage primary liver cancer. ${ }^{12}$ Most common causes of liver failure can be identified and are routinely diagnosed through clinical history, laboratory markers, and radiologic investigations. However, histologic examination of liver biopsies is used for liver transplant. ${ }^{10,13}$ In liver transplant, there are three diagnostic endpoints impacting the patient management or enabling more accurate prognosis that necessitate pretransplant histological examination of the recipient's liver: (1) to confirm the diagnosis of the liver disease and to assess the severity of the disease, (2) to identify concurrent unsuspected pathologies, such as the increasingly common nonalcoholic fatty liver disease (NAFLD), and (3) to obtain diagnostic clues in cases of cryptogenic cirrhosis (even though the occurrence of it is decreasing with increased usage of antiviral treatment). ${ }^{14}$ Histopathological identification of a specific etiology for the liver injury may also allow assessment of the recurrence risk in the allograft and initiation of appropriate therapy to downstage the liver injury to reduce the risk of recurrence of the disease in the allograft. ${ }^{15}$

\subsection{Pretransplant Pathology in Liver Transplant: Assessment of the Donor Liver}

Assessment of the suitability of a donor liver for transplant is a complex information-fusing process incorporating clinical history, laboratory data, gross examination, and histologic diagnosis. The suitability of a donor liver for transplant, or alternatively the risk of a donor liver to the recipient, may be evaluated on two aspects: (1) the risk of poor physiologic function of the allograft and (2) the risk of disease transmission (including viral infection or malignancy) from donor to recipient. A widely used metrics for evaluating the suitability of donors for liver transplant is a donor risk index (DRI) ${ }^{16}$ that has shown to reliably predict graft failure not related to post-transplant infection. ${ }^{17}$ What DRI identifies are clinical criteria and transplant factors that may collectively correlate with poor allograft function. ${ }^{18}$ However, 
DRI does not sample histological factors, some of which may definitively and independently predict the graft survival or longterm functionality post-transplant. For example, macrovesicular steatosis has shown to be an independent risk factor to poor allograft function postreperfusion..$^{19}$ Currently, the only method for diagnosis of macrovesicular steatosis injury in the donor liver is histopathologic assessment. Adequate assessment of the risks of the donor to the physiological function of the recipient thus cannot be achieved in the pretransplant workflow without histopathological examination of the donor liver.

Macrovesicular steatosis, which is graded from 0 to 3 is one of as many as eight types of injuries to a donor liver that are examined routinely during pretransplant workflow by a livertransplant specialty trained histopathologist. The seven other types of liver juries examined during pretransplant pathology workflow are: ${ }^{20}$ microvesicular steatosis that is graded from 0 to 3 , hepatocyte ballooning that is graded from 0 to 3, periportal inflammation that is graded from 0 to 4 , lobular inflammation that is graded from 0 to 4 , portal tract inflammation that is graded from 0 to 4 , necrosis that is graded from 0 to 6 , and fibrosis that is staged from 0 to 6 . These injuries inform differently of post-transplant outcomes. For example, hepatic fibrosis plays a role in long-term allograft survival, particularly when transplanting livers from hepatitis B- or C-positive donors into hepatitis B- or C-positive recipients. ${ }^{21,22}$ Apoptotic hepatocytes and centrilobular necrosis in donor liver were predictors of allograft failure ${ }^{23}$ due to acute cellular rejection following liver transplant. ${ }^{24}$ The post-transplant effects of steatosis are not completely understood. ${ }^{25}$ However, increased level of macrovesicular steatosis in donor liver is currently thought to be associated with greater susceptibility of the allograft to ischemia. ${ }^{26}$ The predictive values of other types of injuries in the donor liver including hepatocyte ballooning and inflammation of various kinds (periportal, lobular, and portal tract) to post-transplant outcomes are less known ${ }^{27,28}$ and likely not as clinically significant as those of fibrosis, necrosis, and steatosis.

\subsection{Paratransplant Pathology in Liver Transplant: Examination of the Explanted Liver}

A histopathologist also plays a key role in examination of the liver explanted from the recipient of liver transplant. ${ }^{29}$ The objectives of histopathologic examination of the explanted liver are to confirm the pretransplant diagnosis, to identify additional comorbidities, and to determine causes of cryptogenic cirrhosis ${ }^{30}$ especially for etiologies that may be heterogeneous and not sampled on pretransplant liver biopsies. ${ }^{31}$ Many ESLDs may also have an increased risk for malignancy. In such patients, it is thus not uncommon to discover clinically undetected early hepatocellular carcinoma or cholangiocarcinoma in the explanted liver, which may have attendant prognostic implications. ${ }^{32}$ For such patients, the pathologic grade or stage of malignancies as well as the histopathological response of the tumor to local therapy also provide important prognostic information for liver transplant. ${ }^{33}$

\subsection{Post-Transplant Pathology in Liver Transplant: Evaluation of the Allograft}

Post-transplant evaluation of the allograft is recognized as the most challenging aspect of liver transplant pathology for managing graft dysfunction. ${ }^{34} \mathrm{~A}$ first challenge to post-transplant pathology is to make various diagnoses of complications that are secondary to rejection or immunosuppression. This challenge is complicated by presence of often overlapping morphological features among the varied etiologies. ${ }^{35}$ A further challenge to post-transplant pathology is to deal with the alteration of classic histologic findings by the immunosuppressed post-transplant state of the patient as well as morphological changes induced by therapeutics administered prior to biopsy. ${ }^{10}$ To make the post-transplant pathology analysis even more intriguing, laboratory findings that would have helped strengthen certain histologic diagnoses before transplant could lose the same complementary significance post-transplant. ${ }^{36}$

\section{Potentiation of a Workpoint for Point-of- Procedure Probing of Optical Biomarkers in Liver Transplant to Enhance Patient Management}

\subsection{Rapidness, Error-Less-Ness, and Artifact-Less- Ness in Diagnostic Yield of Pathology for Liver Transplant}

The technological considerations in the pathology workflow for liver transplant may be appreciated according to the following aspects: (1) the method of acquiring the tissue from the liver (the acquisition aspect), (2) the technique of preparing the tissue specimens for staining (the processing aspect), (3) the yield of diagnosis based on light microscopy examination of the stained specimen (the accuracy aspect), and (4) the duration from acquiring the tissue specimen to yielding the diagnosis (the time aspect). Figure 2 outlines the technology aspects of pathology workflow in liver transplant in association with the robustness of the endpoint for patient management. The largest red check mark (at the rightmost column of the row for paratransplant) indicates the most complete and accurate information that is clinically manageable. The second largest red check mark (at the rightmost column of the row for post-transplant as well as the first row for pretransplant) refers to accurate albeit potentially incomplete information. A half-red check mark (at the rightmost column of the second row for pretransplant) symbolizes practically useful information that may not be entirely accurate.

Any choice of a technology in the clinical workflow is accompanied with a "judicious" element, which determines whether the adoption of the technology will meet, not mitigate, the priorities in the clinical care. Arguably, the first-in-line constraining factor for the choice of technology in the pathology workflow for liver transplant is the turn-around time or "rapidness." Whether the "rapidness" is a concern or not, the diagnostic yield could be subject to sampling error (the "error-lessness") that is associated with the method of tissue sampling allowed for the organ in its clinical setting. When the "rapidness" is a concern, the diagnostic yield is also subject to artifacts (the "artifact-less-ness") that could be introduced by the specimen processing.

The "gold standard" technology for clinical histological analysis of liver specimen is light-microscopy of formalinfixed paraffin embedded (FFPE) hematoxylin and eosin (H\&E) stained tissue sections. ${ }^{20}$ This "gold standard" technology is applied to liver pathology workflow whenever the clinical pathology end-point for patient-management is not constrained by time. Within the pathology workflow of liver transplant, the paratransplant examination of the explanted liver is perhaps least constrained in time. Paratransplant pathology of the explanted 


\section{Pathology work-flow}
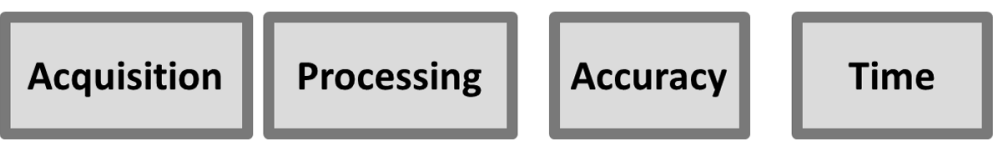

\section{Endpoint}
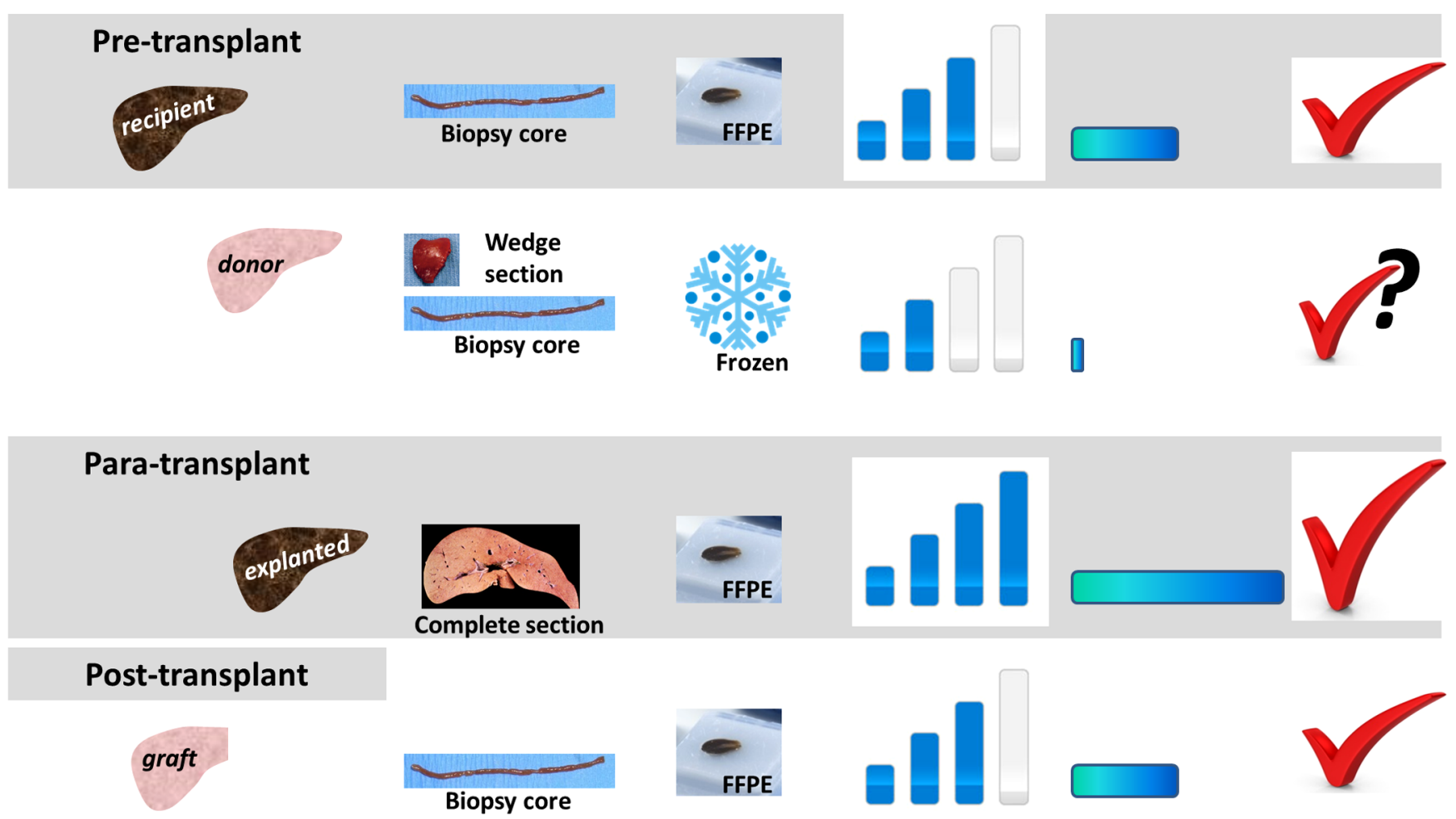

Fig. 2 Pretransplant pathology of the recipient's liver acquires tissue through needle biopsy and processes the core specimens in FFPE for H\&E staining. It may have sampling errors but is free of processing artifacts. The turn-around time is in days but the accuracy is the endpoint for patient management. Pretransplant pathology of the donor liver acquires tissue through wedge section and needle biopsy and processes the wedge and core specimens in frozen section for H\&E staining. It is subject to both sampling errors and artifacts. The turn-around time is $\sim 20 \mathrm{~min}$ as the endpoint is to provide intraprocurement pathology consultation. Paratransplant pathology of the explanted liver acquires tissue through complete sectioning and processes the tissue specimens in FFPE for H\&E staining. It is the most accurate method. The turn-around time is lengthy but irrelevant and the completeness and accuracy is the endpoint. Post-transplant pathology of the allograft acquires tissue through needle biopsy and processes the core specimens in FFPE for H\&E staining. It may have sampling errors but is free of processing artifacts. The turn-around time may be in days but the accuracy is the endpoint for patient management.

liver thus may sample the tissue through complete sectioning and process the tissue specimens in FFPE for H\&E staining. The turn-around time may be lengthy, but it shall yield the needed most accurate analysis of the liver through complete sampling of the organ and specimen processing that maximally preserves the cellular morphology. Within the pathology workflow of liver transplant, the pretransplant pathology of the recipient's liver and post-transplant pathology of the allograft may prefer timely turn-around but nonetheless are not likely to be adversely affected by a diagnosis that is not yielded rapidly. Admittedly, the pretransplant pathology of the recipient's liver and post-transplant pathology of the allograft can only acquire tissue specimens through biopsy, including the assessment at time-zero. ${ }^{23,37}$ But the insensitivity to the turn-around time of the diagnostic yield may allow processing the core biopsy specimens with FFPE for H\&E staining. This pathology workflow for pretransplant diagnosis of the recipient's liver and post-transplant evaluation of the allograft is subject to sampling errors due to biopsy of the large organ but does not suffer from artifacts introduced by the specimen processing. The turnaround time could be at the order of days but the diagnostic accuracy is the pathology endpoint for patient management.

Within the pathology workflow of liver transplant, the pretransplant assessment of the donor liver is where the "rapidness" of rendering the pathology information as well as the comprehensiveness to be rendered by the pathology information dictates a tissue acquisition and specimen processing protocol that has to differ from the rest and may present a point-ofconcern. To assist the procurement surgeon to make the decision of accepting or rejecting a donor organ that will generate timepressing chain actions down the liver-transplant health care system, the current workflow of pathology for donor liver assessment is a "near-the-procedure" approach that turns around the histopathological diagnosis in $\sim 20 \mathrm{~min}$. This turn-around time in the current donor liver pathology workflow is what is necessary for acquiring the tissue specimens from wedge sectioning and biopsy cores, processing the tissue specimens for $\mathrm{H} \& \mathrm{E}$ staining, and making the diagnosis with light microscopy 
when a pathologist is on-duty, whereas the exact occurrence of donor liver availability is often unpredictable.

\subsection{Is There a Potential Workpoint for Injecting New Technology such as point-of-Procedure Optical Biomarkers?}

The significance of the pathological support and the comprehensiveness of the information expected from the histopathological diagnosis for liver transplant leave barely any open call for a new technology, including one that uses light (to the interest of the biomedical optics community) for surrogating any phase or component of the standard pathology workflow. However, there are still undermet or unmet needs in the liver transplant pathology workflow that may await improvements; there are also needs in the liver transplant workflow, where pathology consultation or support could be used but currently is not readily available and which may entail de novo solutions. Room for better clinical management within the pathology workflow for liver transplant resides specifically with pretransplant assessment of the donor liver. The delivery of "rapidness," "error-less-ness," and "artifact-less-ness" in diagnostic yield (READY) by the current near-the-procedure pathology assessment of the donor liver is not ideal. As every minute (with particular concern of warm ischemia) matters in organ quality, donor distribution logistics, and organ transplant, the pretransplant donor liver assessment pursuits "rapidity," which has consequently compromised additional factors of diagnostic yield, particularly the "artifactless-ness" with the current technology available for near-theprocedure pathology assessment of the liver.

The standard histological technique for pretransplant evaluation of the donor liver is the frozen section. A transplant-specialty trained pathologist uses light microscopy to evaluate the morphological histology of a $1-\mathrm{cm}$ wedge sample and two to four needle biopsy cores of the donor liver. The generation and assessment of an $\mathrm{H} \& \mathrm{E}$ stained cryosection takes about $20 \mathrm{~min}$. This standard, near-the-procedure donor liver evaluation technique delivers the "rapidity" that is nonetheless not realtime, is not free of artifacts caused by frozen processing, and has errors due to the sampling of a large organ even with a wedge specimen. In terms of the artifacts, the severity of key histopathological finding can be easily misinterpreted at frozen section. For example, underestimation of steatosis can be caused by air drying or placing the biopsy on a towel or gauze that causes diminishing of fat or fat leaching out of the hepatocyte. ${ }^{38}$ Conversely, overestimation of steatosis can be caused by freezing of water droplets in the tissue when placing the biopsy specimen in saline. ${ }^{10}$ Supplement methods for enhancing visualization of fat are available, such as Oil Red $\mathrm{O},{ }^{39}$ but they lack availability at the time of frozen section and have other artifacts including staining of sinusoids. ${ }^{21}$ When compared with the final complete diagnosis, the overall diagnosis on frozen section of liver has a discordance rate ranging from $1.4 \%$ to $12.9 \% .^{40,41}$ If a significant accumulation of artifacts is present and misinterpreted, they could result in the exclusion of an otherwise acceptable organ or the acceptance of an actually problematic organ.

Intuitively what is more favorable in the pathology workflow for assessment of the donor liver is a "point-of-procedure" approach. A point-of-procedure approach shall generate in real-time the organ information at the organ site, which surrogates the histological yields that are most important to prognosis or ideally the entirety of the histological diagnosis. The point-ofprocedure approach shall also conveniently sample a much

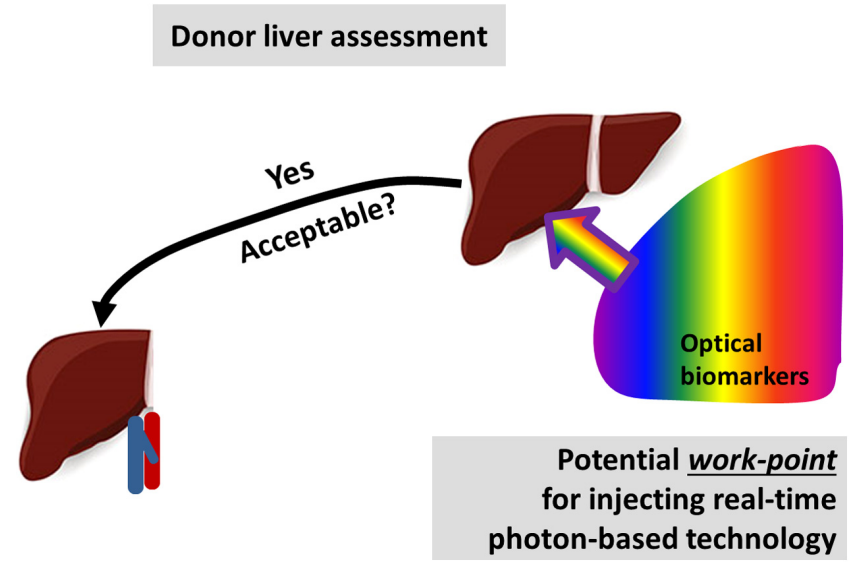

Fig. 3 Technologies for probing of optical biomarkers may be injected to the workflow of pretransplant pathology in liver-transplant to perform point-of-procurement assessment of donor liver suitability. The current clinical workflow of near-the-procurement pathology assessment of donor liver is based on frozen section of one wedge and two to four cores of tissue specimens. This procedure yields diagnosis in $\sim 20 \mathrm{~min}$; however, it is subject to sampling errors of representing the large organ and artifacts caused by the tissue processing technique required for fast turn-around.

greater volume of the donor organ or ideally represent the entire organ to minimize the sampling errors of the information rendered. We postulate that point-of-procedure assessment with the potential of being a surrogate for "near-the-procedure" pathology for injecting into the workflow of pretransplant donor liver assessment may be available with biomedical optics technologies in the capacity of optical biomarkers, as envisioned in Fig. 3. Optical biomarker, for point-of-procedure application, refers to using light (photon) to real-time nondestructively acquire donor organ information that impacts recipient management.

The standard pathology workflow for donor liver evaluation has an additional limitation that might have been under-recognized but the impact could be significant: it identifies morphologically revealed injuries only. Acute or subacute bimolecular or functional injury to the hepatocytes (e.g., that caused by extensive ischemia) that has not yet distorted the morphology but may impact allograft viability over reperfusion is undetectable by routine H\&E tissue sections. ${ }^{42,43}$ The optical biomarkers may have the exact potential to address what routine histopathological information lacks in terms of identifying acute ischemia of the donor liver in the liver transplant workflow. Lack of tools for intraoperative or minimally-invasive assessment of ischemia also has affected management of post-transplant ischemic complications. ${ }^{44,45}$ Optical biomarkers implemented laparoscopically for point-of-procedure operation could be useful for assessing liver injuries including tissue ischemia intraoperatively on a candidate liver, and minimally invasive identification of ischemic site and allograft health during post-transplant management.

\section{Potential of Point-of-Procedure Optical Biomarkers in the Workflow Specific to Liver Transplant}

\subsection{Pathological Consultation is Increasingly Important to Liver Transplant}

Approximately 38,170 people in the USA die of ESLD each year, ${ }^{46}$ making ESLD a leading cause of death in the USA. ${ }^{47}$ 
Additionally, there are $\sim 10$ cases of fulminant hepatic failure per million population ${ }^{48}$ and as high as 80 cases of primary liver cancer per million population in the USA annually. ${ }^{49}$ At this time, liver transplant is accepted as the preferred treatment for patients with ESLD, fulminant hepatic failure, and earlystage primary liver cancer. ${ }^{22}$ However, the available donors are scarce. While $\sim 7100$ liver transplants are performed each year in the USA, ${ }^{50} \sim 16,000$ patients are currently registered on the liver transplant waiting list. ${ }^{50}$ Therefore, attempts have been made to expand the donor pool, including increasing the number of donation after cardiac death (DCD) donors ${ }^{51}$ and using livers considered to be "extended donor criteria." 1,52 As the population ages and more of it develops NAFLD, more livers with "marginal quality" are expected to be seen in potential donors. ${ }^{53}$ Pathologists are called upon to evaluate these livers for transplant suitability, often after-hours or on weekends. $^{21}$

\section{2 "Hard" and "Soft" Pathological Criteria for Rejecting or Accepting a Donor Liver}

The selection of the donor organ influences both the immediate and long-term function of the allograft liver. In rejecting donor liver, there are "hard" pathological criteria that are recommended by all transplant centers. For example, fibrosis greater than portal fibrosis (stage 2 or greater in the Batts and Ludwig and Ishak-Knodell classifications ${ }^{54}$ ) is among the "hard" thus absolute contradictions to transplant. In accepting donor liver, however, there are "soft" pathological and clinical criteria that are practiced differently across transplant programs. One issue being debated is whether livers with less severe forms of macrovesicular steatosis are acceptable for transplant. ${ }^{52,55}$ Typically, donor livers that have greater than $30 \%$ macrovesicular steatosis should be deferred, but the decision is ultimately left to the transplant surgeon. ${ }^{56}$ For necrosis, there is no consensus for the amount that is acceptable ${ }^{57}$ although a cutoff of $10 \%$ diffuse necrosis (ignoring focal subcapsular necrosis) has been suggested. ${ }^{21}$ Another "soft" medical criterion practiced with wide disparity from center to center and from patient to patient is the warm ischemia time for DCD. ${ }^{58}$ The lack of consensus on the warm ischemia time reflects the particularly pressing need of a real-time point-of-procedure device/method that can assess the ischemia or ischemic damage to the donor liver. A real-time point-of-procedure device/method that can assess the ischemia or ischemic damage to the donor liver will also be useful to realtime intraoperative assessment of the ischemia or ischemic site post-transplant, should the device allow laparoscopic probing of the tissue site.

\subsection{Undermet and Unmet Needs in the Pathology Workflow for Donor Liver Assessment}

Currently, there is an undermet need in donor liver assessment for intraoperative (point-of-procedure), real-time, and nondestructive assessment of the donor liver injuries that are the most significant predictors of post-transplant viability. There is also an unmet need in donor liver assessment for real-time and nondestructive identification of ischemia or irreversible injuries to the donor liver earlier than appearing on morphological histology examined with light microscopy of H\&E stained specimens. We anticipate that biomedical optics technologies convenient for nondestructive evaluation of tissue interior and functional constituents, including one of the simplest forms as diffuse optical spectroscopy (DOS), or diffuse reflectance spectroscopy (DRS), have the potential of probing optical biomarkers for point-of-procedure, real-time surface assessment of parenchymal injuries of donor liver to assist the decision-making regarding transplant suitability. The well-known real-time robustness of light-based technology on assessing ischemia may also render the exact capabilities that are lacking in the current pathology workflow in the pretransplant setting or the post-transplant patient management.

\subsection{Potential and Pitfalls of Point-of-Procedure Optical Biomarkers Toward Liver Transplant Manifested by Photonic-Identification of Steatosis}

The potential of light-based technology for generating tissue information that may surrogate a portion of histopathological diagnosis has been recognized since Jobsis ${ }^{59}$ demonstrated that spectrally remarkable and functionally important tissue constituents including hemoglobin of various states could be probed with light in the visible and near-infrared spectra. The optical spectral sensitivity to scattering also allows interrogating the changes (such as the collagen matrix) of architecture at the subcellular level. These fundamentally robust capacities of light-based technology that can also be applied in nondestructive means have been tested for noninvasive diagnosis, ${ }^{60-62}$ adapted to monitoring of tissue responses to therapy, ${ }^{63-65}$ and facilitated toward surgery planning. ${ }^{66-69}$ Photon-based techniques are particularly suitable for organ-site application ${ }^{70}$ to assess the spectral constituents of an object because photon illumination and collection can be conveniently configured on the tissue surface with the use of fibers or remotely with optics. ${ }^{71}$ All light-based technologies can share the features of noninvasiveness (portability, real-time response, multifunctionality, operational convenience, etc.) that, when combined, could potentiate optical biomarker probing for point-of-procurement assessment of donor liver. One simple technique that merits considerations for point-of-procedure probing of optical biomarkers is DOS/DRS, one of the simplest forms in which light-based technology can be configured for organ-site application. Laparoscopic implementation of light-based technologies such as DOS/DRS ${ }^{72}$ may also render the utility for posttransplant in-situ pathology management, when identification of optical biomarkers in the conventional opened tissue-probing setting has been established.

To our knowledge, there are just a handful of studies on lightbased technologies with relevance to the liver transplant setting, which were estalished in vivo or based upon surface measurement. These studies include some for identification of steatosis in animal models or human specimens and some others for identification of fibrosis in animal models. Reports of light-based technologies for identification of pathologies in livers other than steatosis and fibrosis, including necrosis, inflammations of various types, and hepatocyte ballooning with the application relevancy to liver transplant, are scarce. The convenience of tissue interrogation as well as the information richness inherent to the spectral probing has made DOS/DRS a common choice among those earlier studies demonstrating the relevancy to liver transplant. In the following, we review some reports of lightbased identification of steatosis. Light-based identification of liver injuries other than steatosis in a transplant setting may be limited to fibrosis only and will be briefly overviewed in the section addressing the effect of the liver capsule on probing of optical biomarkers. 
Liver steatosis is graded according to a semiquantitative metrics: $:^{73}$ a grade of 0 or "absent" for $<5 \%$ hepatocytes affected by lipid infiltration, 1 or "mild" for $5 \%$ to $<30 \%$ infiltration, 2 or "moderate" for $30 \%$ to $<60 \%$ infiltration, and 3 or "severe" for $\geq 60 \%$ infiltration. Between the two subtypes of steatosis, microvesicular steatosis is not associated with primary graft nonfunction after transplant, ${ }^{74}$ whereas macrovesicular steatosis is a known risk factor to ischemic reperfusion injury ${ }^{19}$ post-transplant. Macrovesicular steatosis is also reported to cause adverse outcomes in living donors who undergo right hepatectomy. ${ }^{75}$ With the shortage of donor organs, livers with mild levels of steatosis, and preferrably morphometrically microvesicular type of steatosis, ${ }^{76}$ are considered for transplant. Gross assessment of the liver by visual inspection and palpation has shown low accuracy of identifying the parenchyma steatosis. ${ }^{52}$ Conventional radiological imaging modalities, such as computed tomography and magnetic resonance imaging/spectroscopy, are logistically difficult for procurement setting even though they provide highly accurate assessment of the global steatosis. ${ }^{77}$ The sonographic hepatorenal index ${ }^{78}$ allows bedside ultrasound (US) imaging to assess steatosis but lacks the sensitivity to reliably identify livers with mild level of steatosis. ${ }^{79}$

Optical biomarker-based identification of steatosis of liver will face three major challenges: (1) the ability to detect mild level of steatosis, (2) the ability to differentiate macrovesicular steatosis from microvesicular steatosis at the absence of other pathologies, and (3) the ability to discriminate steatosis in the presence of other pathologies. The detection of mild level of steatosis is a matter of sensitivity of a light-based technology that responds to fatty deposition in liver tissue. The differentiation of macrovesicular steatosis from microvesicular steatosis in the absence of other pathologies is an issue of microscopic specificity of a light-based detection method regarding how fatty vacuole of different size distribution gives rise to different signal patterns when probed by light. The discrimination of steatosis in the presence of other pathologies is a challenge of the macroscopic specificity that is also influenced by the sensitivity of the light-based technology on detecting fatty changes. In reality, the challenge of light-based technology showing the potential for probing optical biomarkers of steatosis is to detect mild level of steatosis, and to discriminate macrovesicular steatosis from microvesicular steatosis, in the presence of other pathologies. Identifying the grade or stage of a particular liver injury including steatosis to inform the acceptance or rejection, in the presence of other pathologies, is a common challenge to any light-based pathology aimed for assessing a type of liver injury predictive of transplant outcomes.

Several studies supporting photonic identification of steatosis in liver, using primarily DOS or DRS, have been reported. Evers et al. ${ }^{80}$ and Westerkamp et al. ${ }^{81}$ sampled the strong lipid absorption around $1210 \mathrm{~nm}$ using DRS over a broad visiblenear-infrared spectrum up to $1600 \mathrm{~nm}$ for identifying hepatic steatosis in human liver specimen or during the surgery for measuring within the liver parenchyma. However, the prominent absorption of lipid at $1210 \mathrm{~nm}$ has limited sensitivity and specificity when detecting mild level of steatosis. ${ }^{80,82}$ Furthermore, whether it is micro- or macrovesicular steatosis is not to be informed by absorption alone, there is no known report of light absorption differing between macrovesicular steatosis and mirovesicular steatosis. A time-resolved near-infrared spectroscopy study by Kitai et al. ${ }^{83}$ on rat liver specimens suggested that the fat droplets inside the hepatocytes were the dominant scatterers over the visible to near-infrared spectrum. That generated significant interest in using scattering spectral changes to assess steatosis. Surface measurements on human liver specimens by McLaughlin et al. ${ }^{84}$ using DRS over 550 to $1040 \mathrm{~nm}$ showed a correlation between the diffuse reflectance intensity with the histological lipid mass fraction of liver tissue. Evers et al. ${ }^{80}$ have also hypothesized that lipid infiltration changes the scattering power, which indicates the scattering spectral-dependence that may inform particle size information useful to assessing the primary subtype of the steatosis. Evers's hypothesis was supported by a recent study ${ }^{85}$ that by directly sampling the liver parenchyma during the longitudinal steatosis development has discovered that the fatty changes of livers caused the elevation of the scattering power at the early stage of hepatic steatosis development. The changes of the lipid size at the early stage of the steatosis evaluated on the histopathological imagery also provided morphometric evidence of the changes of the scattering power that could be resolved from the optical spectral measurements. ${ }^{86}$ The study may have demonstrated the potential of detecting a mild level of steatosis using light-based technology, but to differentiate macrovesicular steatosis from microvesicular steatosis, even at the absence of other pathologies is challenging as no specific spectral feature is known that differs between macrovesicular type lipid (large droplets of the size of hepatocytes) and microvesicular type lipid (clusters of granular droplets much smaller than hepatocytes). To discriminate steatosis in the presence of other pathologies is to be further confounded by the need to isolate steatosis features from the composite features corresponding to other pathologies when they present.

\section{Overarching Challenges of Optical Biomarkers in Liver Transplant}

Light-based technology could serve among the obvious choices for alleviating or even solving the deficiencies on "READY" of the current near-the-procedure pathology workflow for donor liver assessment and other aspects of pathology consultation needed in liver transplant. There is a plethora of biomedical optics technologies or light-based technologies that have been injected into clinical diagnosis. Any technology for injecting into and thus eventually becoming part of the clinical workflow must have uniquely strong benefits that will outweigh the recognized drawbacks of the current approach with little or no compromise to the workflow for patient management. The light microscopy, as it is the gold standard technology of clinical histology, is not a part of light-based diagnostic technologies to be discussed in the following.

\subsection{Which Traits of Light-Based Technology May be Viable for Point-of-Procedure Optical Biomarkers?}

All light-based technologies applying to an organ for in situ diagnostic yield can, in principle, operate in real-time; therefore, all of light-based technologies can become candidates for pointof-procedure probing of optical biomarkers, but some may have stronger potential than others for clinical translation. The light-based diagnostic technologies are generally separated into two major types: (1) those that provide morphological details and (2) those that provide functional contrasts. There are also some technologies that may deliver a combination of both morphological details and contrasts. Imaging the morphology of deeper tissue (deeper than the thickest layer of specimens 
presented to a histopathologist) with the resolution fine enough to allow histological level information is fundamentally challenged by light scattering, whether the tissue is to be probed invasively or noninvasively. For noninvasive imaging of shallow tissue or superficial layer, significant advancements have been made to improve the spatial resolution of photon-based morphological imaging modalities to super-resolution level. However, it may be overly bold to anticipate that a morphological-imaging technology will be able to surpass the combination of spatial resolution, contrast detail, field-of-view, and convenience of light microscopy, upon which a histopathologist depends on a daily basis, when a specimen is made available routinely even at the compromise of a short lead time. In that sense, a biomedical optics technology that renders solely or primarily morphological information for in situ structural detailing of the organ is unlikely to motivate surrogating the conventional routine of histological assessment of donor liver morphology on specimen. What may better motivate adoption of point-ofprocedure technology into the pretransplant workflow for donor-liver assessment, as the call of which has to be made by the procurement surgeon, is the yielding of the diagnostic information that is not only as robust as the part of conventional pathology yield that accurately impacts patient management, but also facilitated in real-time and at the organ site to allow point-of-procedure decision-making. What may motivate an even more enthusiastic treatment of point-of-procedure optical biomarker technology within the pretransplant workflow for donor-liver assessment or other time-points of patient management is the addition of tissue information such as ischemia, which is not readily assessable with the conventional pathology workflow or difficult to sample from the patient.

Whether a type of contrast-yielding optical biomarker can motivate the initial investigation, and if it survives then the subsequent clinical translation to liver transplant may be governed by the following two overarching questions: (1) do the pathologies of liver important to post-transplant patient management generate optical contrast? and (2) if a liver pathology important to posttransplant patient management does present a contrast for optical probing, how sensitive and specific is the optically probed contrast? Only when a morphological abnormality of the liver that is predictive of post-transplant outcome presents a contrast that can be sensitively and specifically acquired by optical probing of tissue can the optical biomarker be translated. Unfortunately, the information we have at the present time regarding either of the two overarching questions is quite limited. The limited knowledge regarding both questions has limited the initiation of technology development for optical biomarker endpoints. Conversely, the knowledge regarding both questions is also limited by the unavailability of the technology suitable for, or explored toward, optical biomarker identification.

\subsection{Capsule Will Confound All Light-Based Technologies Toward Point-of-Procedure Optical Biomarkers}

The capsule is a thin collagen-rich layer enclosing a solid organ like the liver or kidney, which are among the most transplanted organs. The capsule is negligible at the macroscopic operation and usually ignored by conventional histology examination. The capsule, however, may confound all approaches of the surface assessment that is imperative to point-of-procedure probing of optical biomarkers of the parenchyma. That is because the capsule that differs morphologically from the subcapsular parenchyma also differs from the subcapsular parenchyma in optical properties, most pronouncedly in its high level of scattering due to collagen, ${ }^{87}$ which will affect every optical biomarker approach intended for assessing the subcapsular parenchyma in situ, regardless of the spatial or spectral information that is being extracted.

Capsule of kidney, the most transplanted solid organ, is welldocumented, by optical coherence tomography imaging, ${ }^{88}$ as a thick layer of high scattering intensity. The highly scattering kidney capsule may cast a shadow of light probing of the subcapsular parenchyma, reducing the sensitivity, and worsening the spatial resolution for visualizing a morphological feature in the subcapsular parenchyma. The highly scattering kidney capsule will also disguise the spectral characteristics of the subcapsular parenchyma. The effect of the kidney capsule on surface spectral measurement of subcapsular parenchyma could have been noticed before but has not been attended to and understood quantitatively until recently. ${ }^{89,90}$ A liver's capsule is comparatively very thin, $\sim 10 \mu \mathrm{m},{ }^{91}$ but its effect on surface assessment of liver parenchyma cannot be overlooked when optical biomarker is concerned. Nilsson et al. ${ }^{82}$ have projected that surface DRS measurement of liver represents the DRS on the cut-section of the liver, based on intraoperative and ex vivo measurements on both the surface and cross-section of human livers covering a wide spectral range of 450 to $1550 \mathrm{~nm}$. Between the DRS on the surface of liver in the presence of the capsule, and that on the cross-section of liver, the profiles were nearly identical over 1000 to $1550 \mathrm{~nm}$. However, there was a small albeit consistent elevation of the DRS over the 600 to $1000 \mathrm{~nm}$ range between that performed on the surface of liver in the presence of the capsule compared to that on the cross-section of liver. When the liver contained a tumor, the deviation between the DRS on the surface of liver in the presence of the capsule and the DRS on the cross-section of the tumor was significantly different over the 400- to 1100 $\mathrm{nm}$ spectral range. These observations implied that even a capsule of macroscopic insignificance as thin as that of liver would affect surface spectral assessment of pathological changes in the parenchyma. Spectral optical biomarkers, or determination of the parenchyma chromophore components or scattering parameters, thus have to depend on the knowledge of the capsular effect in order to isolate the spectral characteristics true to the subcapsular parenchyma. For a thicker capsule such as that of kidney of $\sim 200 \mu \mathrm{m}^{88}$ which is much thicker than that of liver, the now-known capsule effect upon surface spectral measurement has been shown to be more pronounced. ${ }^{90}$ In measurement of renal ischemia during laparoscopic partial nephrectomy ${ }^{92}$ over a spectral response of 500 to $650 \mathrm{~nm}$, DOS measured on the capsular surface of kidney underestimated $\sim 30 \%$ of deoxyhemoglobin at $550 \mathrm{~nm}$ when the capsule effect was not taken into account in the inverse model.

Our recent measurements from livers and kidneys are useful to appreciating how much the capsule could affect surface measured spectral profile, ${ }^{90}$ as shown by the compiled results in Fig. 4. The results correspond to DOS performed on 10 fresh human livers ex vivo and 9 fresh human kidneys ex vivo [University of Oklahoma Health Sciences Center (OUHSC) IRB \#8155] using a surface probe with a 3-mm source-detector separation. The panels (a) and (b) outline conceptually how diffuse reflectance of a subcapsular medium is affected by the thickness of the capsule. In panel (c), the thinner solid line corresponds to the average of 50 DOS measurements on 


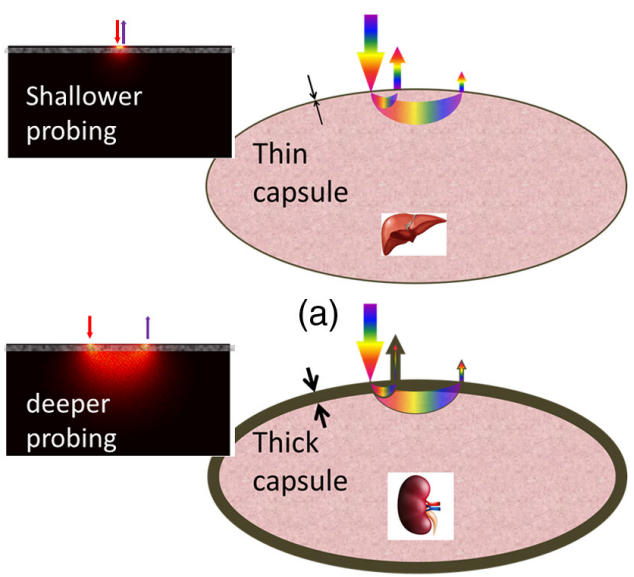

(b)

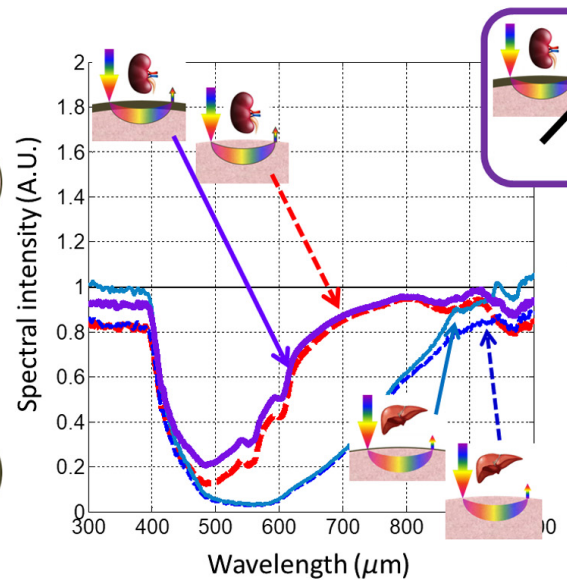

(c)

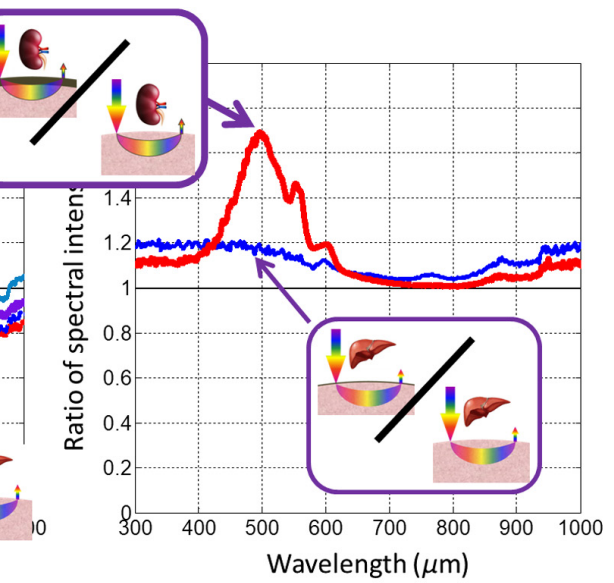

(d)

Fig. 4 (a) The effect of a thin (liver) capsule on surface DOS at two source-detector separations. (b) The effect of a thick (kidney) capsule on DOS at two source-detector separations. Between panels (a) and (b), a thicker capsule or a capsule having a stronger optical contrast over the subcapsular tissue will affect more of the surface DOS. (c) Measured capsular effect: kidney (thicker lines) versus liver (thinner lines), and surface (solid lines) versus subcapsular/cross-sectional (broken lines). (d) The thicker solid line is the ratio between surface DOS and subcapsular DOS averaged for 9 kidneys, and the thinner line is the ratio between surface DOS and cross-sectional DOS averaged for 10 livers. Data compiled from Ref. 90.

the capsular surface of 10 livers. The thicker broken line corresponds to the average of 50 DOS measurements on the cutsection of 10 livers. The thicker solid line corresponds to the average of 45 DOS measurements on the capsular surface of nine kidneys. The thinner broken line corresponds to the average of 45 DOS measurements on subcapsular (cortex) surface of nine kidneys. In panel (d), the thinner solid line shows the spectral ratio of the DRS measured on the capsular surface over that on the cut-section of liver. The thicker solid line shows the spectral ratio between the DRS measured on the capsular surface over that on the cortex surface of kidney. A spectrally flat line at " 1 " would indicate that the capsule does not affect surface measurement of the spectral characteristics of the subcapsular parenchyma (by DOS). Apparently, the thicker the capsule (optically heterogeneous with respect to the subcapsular tissue) is (typical thickness: kidney $200 \mu \mathrm{m}$ and liver $10 \mu \mathrm{m}$ ), the stronger the capsular effect is to the surface assessment of the spectral characteristics of the subcapsular parenchyma. ${ }^{92}$ The liver capsular effect is relatively small ${ }^{82}$ but apparently not negligible. The surface DOS of liver parenchyma deviates from the DOS of parenchyma alone $5 \%$ to $20 \%$. Not correcting the spectral variation due to the capsular effect will cause the parenchymal spectral properties to be resolved inaccurately and that will undermine any potential for identifying optical biomarkers.

The collagen content of the capsule has actually been suggested as an indicator of the parenchymal fibrosis by some light-based modalities performed on the surface of liver in rodent models ${ }^{91}$ that also correlated the thickness of the capsule with the parenchymal fibrosis. However, the increase of liver capsule thickness (detecting it is quite feasible with optical coherence tomography) associated with the fibrosis development ${ }^{91,93}$ may not be true in the presence of multiple pathologies. We have measured capsule thickness on 12 of 16 livers unaccepted to transplant but did not find increased capsular thickness correlating with increased parenchymal fibrosis (not published). Our initial results were probably unreliable due to the small sample size; however, one needs to be cautious in associating the increase of the liver capsule thickness with higher fibrosis stage. Probing the fibrosis of the capsule alone with optical biomarkers may misrepresent the parenchymal stage of fibrosis in the presence of other pathologies. Probing the fibrosis content in the parenchyma as a more direct assessment of the fibrosis without any influences of the capsular shielding effect has been evaluated with DOS on FFPE samples. ${ }^{94}$ The study implemented a fibrosis stage of 0-4 in comparison to the 0-6 that is routine to liver transplant pathology. The study has reported that the DOS signal corresponding to fibrotic liver was higher than the DOS signal of none-fibrotic livers and that could attribute to the increase of tissue scattering by the increase of the hepatic collagen content. However, the study finding of lower DOS signal at higher ( 3 and 4 at a scale of 4) fibrosis stage than lower fibrosis stage (1 and 2 at a scale of 4) was perplexing.

\subsection{Homogeneity of the Optical Biomarker Contrast Over the Volume of the Donor Organ}

Surface spectral measurement for optical biomarkers will acquire the spectral remission from the medium surface to assess the spectral alterations caused by tissue spectral heterogeneities within the volume of sampling. How sensitive or reliable an optical biomarker technique is to the tissue pathology that alters spectral features is to be affected by both the spectral contrast of the tissue pathology over the baseline (normal) tissue and the spatial homogeneity of the optical biomarker contrast over the organ. No tissue pathology may be microscopically homogeneous. However, a spectral assessment may only need to interrogate a volume of tissue with the tissue pathology that is mesoscopically homogeneous. As the spectral alterations are caused by the microscopic parenchymal heterogeneities in absorption and/or scattering that are secondary to changes in biochemical function or pathology, sensing the lumped optical properties of the tissue over a mesoscopic scale by using light diffusely traversing through the volume of tissue will be useful. 


\section{Benchmarks, Pitfalls, and End-Points toward Translation}

There are some benchmarks that any candidate technology of optical biomarkers must demonstrate in order to motivate clinical interests: (1) the sensitivity and specificity of the technology in identifying each type of liver injury in the presence of mixed pathologies (the information accuracy aspect); (2) the short-term and long-term predictabilities of the optical biomarker of liver injury to post-transplant organ viability (the patient management aspect); and (3) the simplicity, reliability, and the intuitiveness of the technology to an end-user (the workflow/field-applicability aspect). Among these three aspects, the second one is uniquely challenging to establish, because between a morphological deformation and a detectable optical feature, there lies in a tissue-light interaction mechanism that has to be understood adequately. The predictability of a histologically identified liver injury to post-transplant organ viability thus does not translate directly to the predictability of the optical biomarker of the same liver injury. An optical biomarker, however, may enable "magnifying" a histologically identified liver injury with a detectable optical feature, which would then allow diagnosis of liver injury at a level earlier than identified by histopathology and grading a liver injury at a level finer than currently classified by histopathology. It is also quite possible that no optical biomarker is identifiable for certain types of liver injury.

The potential of translating an optical biomarker technology for point-of-procedure implementation will certainly be determined at first by the robustness of the diagnostic information offered to surrogate the histopathological yield that is most relevant to post-transplant outcomes. The pitfalls for translating any optical biomarker technology for point-of-procedure adoption in a procurement setting, or in its extension to post-transplant setting, however, will appear first with the form-factors, such as the device applicator, and how the information is presented to the end-user at the site of operation. An optical biomarker technology that is delivered as a simple approach to realtime, nondestructive assessment of donor liver pathologies will find a less treacherous path to translation than one that imposes even slightly longer learning curve. As prospected in Fig. 5, a simplest form of the device for the end user (a procurement surgeon) at the point of procurement is preferably operated with a hand-held applicator probe that can be placed at any site of the liver surface for stationary or free-hand scanned surface assessment of the liver, regardless of the type of light-based technology. Whether the device offers remote assessment is not much of a concern in assessing donor liver, and the removal of any artifacts caused by remote assessment by using a contact probe will probably be more important than the appeal of a remote operation on the donor organ. Additionally, a device for real-time nondestructive assessment of liver pathology may not have much merit for translation if it does not generate indicators of multiple pathological conditions of a donor liver, including, for example, fibrosis, necrosis, and steatosis. Whichever the optical signature of each condition of these pathologies is,

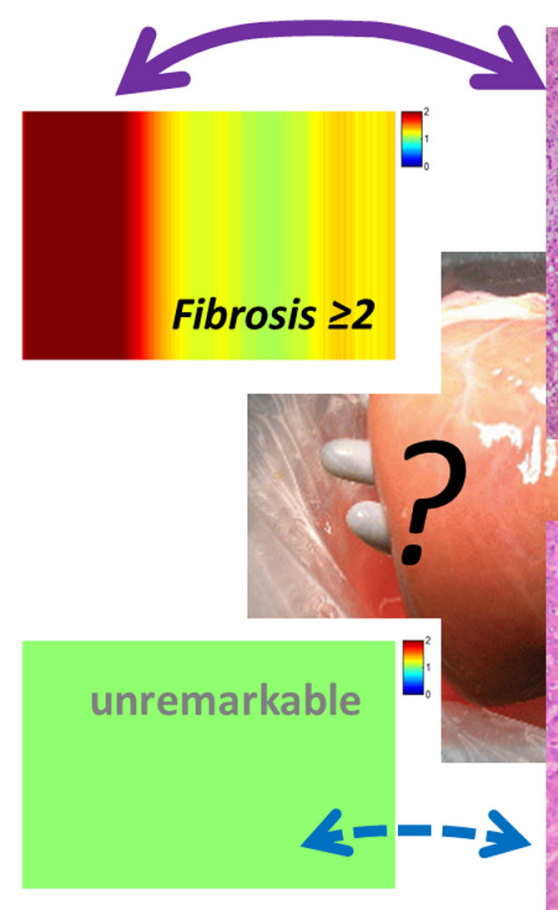

(a)

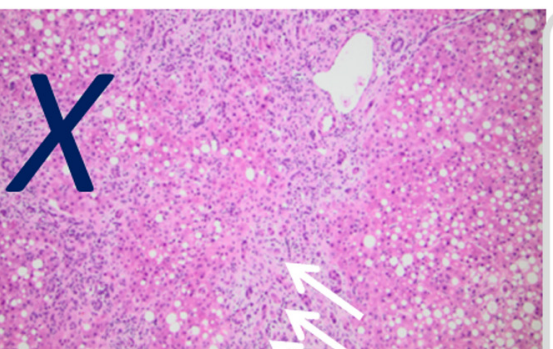
2.
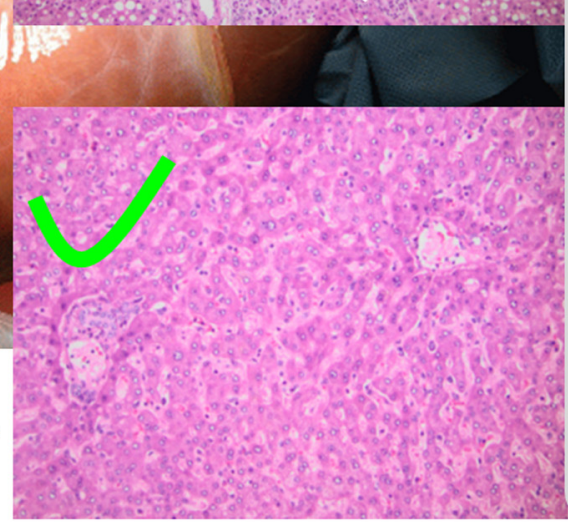

(b)

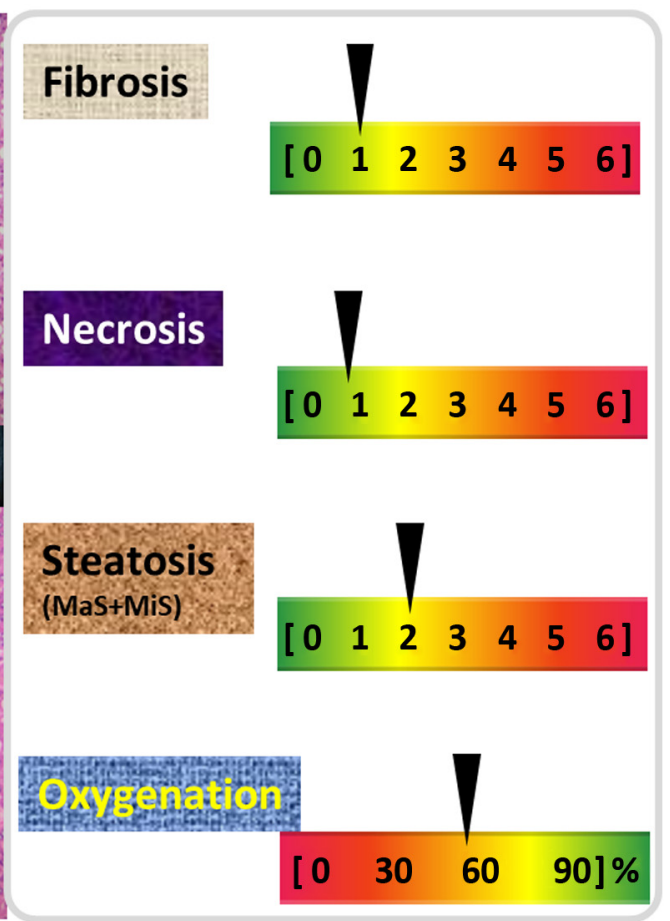

(c)

Fig. 5 The prospect of nondestructive surface assessment of liver pathologies most predictive of posttransplant outcomes. (a) Any point-of-procedure optical marker information is preferably rendered as an image that could "visualize" the presence of an abnormal or unacceptable parenchymal condition at the site of nondestructive surface assessment. (b) The surface "visualization" given in (a) can be associated with the parenchymal pathology, such as one with unacceptable stage of fibrosis (upper histology image, fibrosis marked by the three arrows) or one that is morphologically normal (lower histology image). The information rendered to the end-user by point-of-procedure probing of optical biomarkers could also be converted to scaled presentations shown for: fibrosis staged within [0-6], necrosis graded within [0-6], steatosis graded within [0-6] (MaS+MiS), and oxygenation quantified within [0-90]\%. 


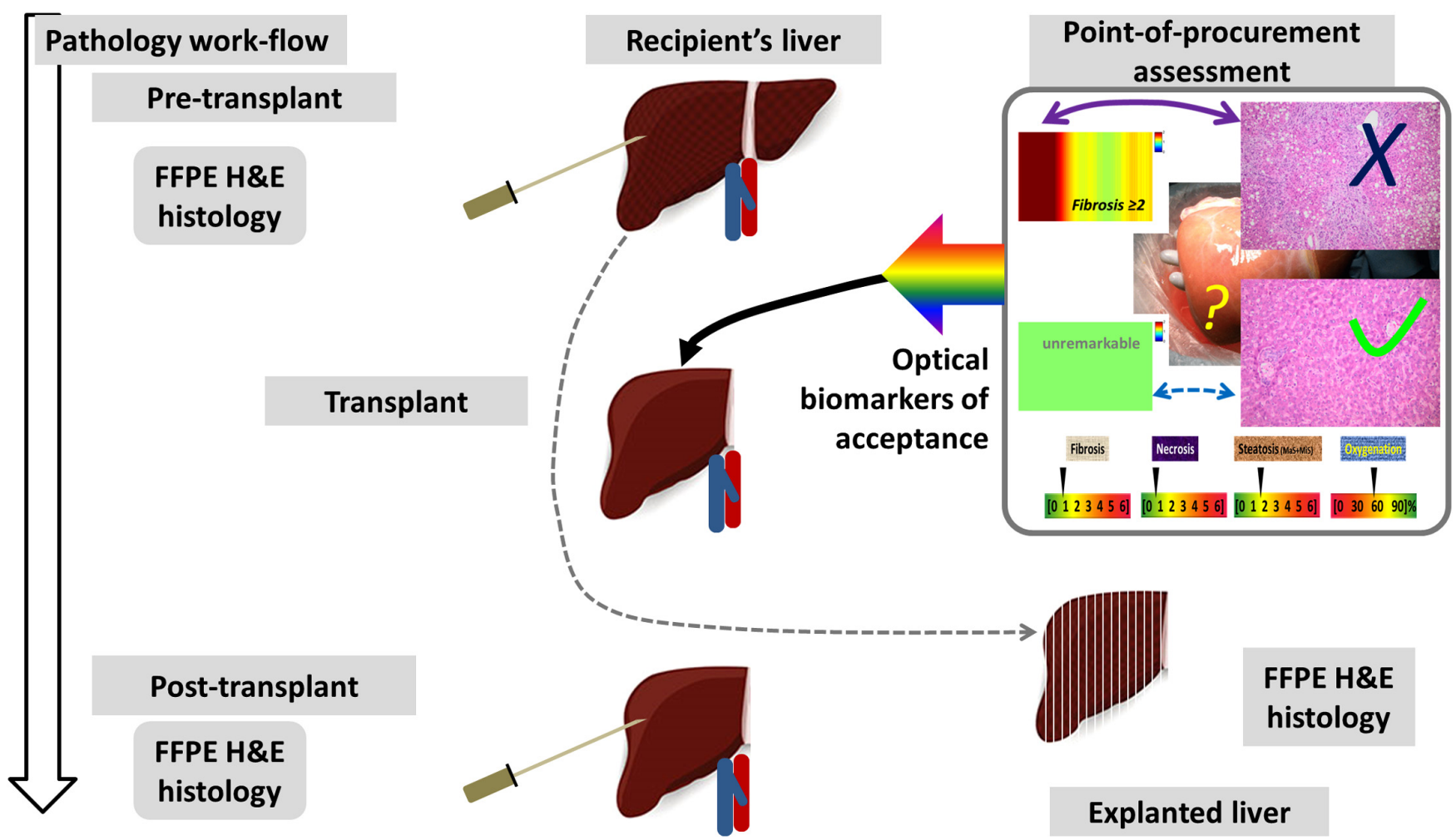

Fig. 6 A projection of pathology workflow with the injection of point-of-procedure optical biomarkers is possible in the pretransplant assessment of donor liver. Pretransplant assessment of the donor liver at a procurement point can potentially be performed nondestructively by surface measurements on the exposed liver for probing of optical biomarkers. The imagery and tissue features corresponding to a few significant pathological endpoints are delivered in real-time as the probe is scanned over the entire surface of the donor.

the optical diagnostic yield will be appreciated more straightforwardly if they are displayed as images such as those conceptualized in Fig. 5(a), encouraging intuitive assessment of the extensiveness of the pathology, and correlated to the qualitatively abnormal features shown on the optical signals. Quantitative assessment may also be possible by displaying the optical diagnostic yields with quantitative scales, such as those envisioned in Fig. 5(c), that inform fibrosis stage of $0-6$, necrosis grade of $0-6$, and steatosis grade of $0-6$ (when macrovesicular steatosis and microvesicular steatosis cannot be distinguished so the scales are summed). With the ability to discriminate between macrovesicular steatosis and microsteatosis, two separate meter scales may be used, respectively, for macrovesicular steatosis and microvesicualr steatosis to inform the grade of 0-3 for each. Perhaps, an addition of optical biomarkers to the point-of-procedure assessment of donor liver which is not available currently is the parenchymal oxygenation scaled over approximately [0-90]\% or other properties informing tissue health. Knowledge of tissue oxygenation or biochemical level tissue health could help understand the extent of donor injury due to ischemia, and to ultimately develop a "hard" criterion for managing donor organs procured after unfavorable or uncertain length of warm ischemia.

An end-figure of translation of point-of-procedure optical biomarkers is likely one that is illustrated in Fig. 6. Injecting point-of-procedure optical biomarkers into pathology workflow is most likely possible in the pretransplant assessment of donor liver. Pretransplant assessment of the donor liver at a procurement point can potentially be performed nondestructively by surface measurements on the exposed liver through a handheld probe for scanning over the entire surface of the organ for optical biomarkers identification. The optical biomarker information, including imagery and meter-scales featuring tissue conditions that may be most predictive of post-transplant outcomes, has to be delivered in real-time as the probe is scanned over the entire surface of the donor for point-of-procedure decision making.

It must be acknowledged that our discussion of the potential of point-of-procedure optical biomarkers is quite limited since some of the translational aspects are apparently restricted to a relatively simple technology of DOS, with which the authors have had direct experiences relevant to liver transplant. However, the pitfalls hindering clinical translation of a candidate technology that is as simple as DOS have broad implications to any candidacy technology of optical biomarkers for point-ofprocedure implementation. It must also be noted that any potential of "point-of-procedure optical biomarkers" in the transplant regimen projected for one organ type may not translate directly or unaltered when applying to another organ type, because the pathology consultation or support in pretransplant and posttransplant managements has significantly diverse clinical roles across transplant of different types of solid organ.

\section{Conclusions}

There is not much historical basis in solid-organ transplant for diagnostic or therapeutic applications of the rapidly growing translational biomedical optics technologies. However, there are concerns in the pathology workflow of solid-organ transplant 
and under-recognized deficiencies that present unique opportunities for injecting biomedical optics technologies. A pressing area of biomedical optics in the pathology for liver transplant is the discovery of optical biomarkers for using light to develop "point-of-procedure" objective organ information useful to patient management and prognosis. Point-of-procedure optical biomarkers of organ injuries, such as steatosis and fibrosis and detrimental conditions, such as ischemia, will have direct implications for patient management in pretransplant stages. The potential of point-of-procedure optical biomarkers toward clinical translation may also be extended to post-transplant management if the pitfalls are effectively overcome.

\section{Disclosures}

The authors declare no competing financial interests.

\section{Acknowledgments}

The authors thank Halen Borron, a medical student in OUHSC, and LifeShare, Oklahoma City, Oklahoma for assisting some data acquisitions. The contribution of E.M.R. to this work is supported in part by the James Park Dewer, MD, professorship of pathology in the Department of Pathology at OUHSC.

\section{References}

1. A. Rana et al., "Survival benefit of solid-organ transplant in the United States," JAMA Surg. 150(3), 252-259 (2015).

2. R. F. Saidi and S. K. Hejazii Kenari, "Challenges of organ shortage for transplantation: solutions and opportunities," Int. J. Organ. Transplant Med. 5(3), 87-96 (2014).

3. P. Kefalides, "Solid organ transplantation. 2: Ethical considerations," Ann. Intern. Med. 130(2), 169-170 (1999).

4. U.S. Health Resources and Services Administration, "United network for organ sharing; policies and rationale for transplantation of organs for foreign nationals and exportation of organs outside the United States; general notice," Fed. Regist. 53(53), 8977-8978 (1988).

5. Council on Scientific Affairs, "Introduction to the management of immunosuppression," J. Am. Med. Assoc. 257(13), 1781-1785 (1987).

6. K. Suzuki et al., "Quantitative measurement of optical parameters in the breast using time-resolved spectroscopy. Phantom and preliminary in vivo results," Invest. Radiol. 29(4), 410-414 (1994).

7. B. W. Pogue et al., "Fluorescence-guided surgery and intervention-an AAPM emerging technology blue paper," Med. Phys. 45(6), 2681-2688 (2018).

8. G. Duvoisin et al., "Novel biomarkers and the future potential of biomarkers in inflammatory bowel disease," Mediators Inflamm. 2017, 1936315 (2017).

9. J. M. Carethers, J. Braun, and B. E. Sands, "Genetics, genetic testing, and biomarkers of digestive diseases," Gastroenterology 149(5), 11311133 (2015).

10. B. Geramizadeh and S. A. Malek-Hosseini, "Role of histopathologist in liver transplantation," Int. J. Organ. Transplant. Med. 8(1), 1-6 (2017).

11. J. R. Lilly et al., "Auxiliary liver transplantation in acute liver failure," J. Pediatr. Surg. 7(5), 492-498 (1972).

12. R. W. Laing et al., "Viability testing and transplantation of marginal livers (VITTAL) using normothermic machine perfusion: study protocol for an open-label, non-randomised, prospective, single-arm trial," BMJ Open 7(11), e017733 (2017).

13. C. T. Wai et al., "Liver transplantation for hepatocellular carcinoma in Singapore," Singapore Med. J. 47(7), 584-587 (2006).

14. P. J. Thuluvath, S. Hanish, and Y. Savva, "Liver transplantation in cryptogenic cirrhosis: outcome comparisons between NASH, alcoholic, and AIH cirrhosis," Transplantation 102(4), 656-663 (2018).

15. N. Faisal and E. L. Renner, "Recurrence of autoimmune liver diseases after liver transplantation," World J. Hepatol. 7(29), 2896-2905 (2015).

16. S. Feng et al., "Characteristics associated with liver graft failure: the concept of a donor risk index," Am. J. Transplant. 6(4), 783-790 (2006).
17. L. H. Rosenberger et al., "Donor risk index predicts graft failure reliably but not post-transplant infections," Surg. Infect. (Larchmt) 15(2), 94-98 (2014).

18. D. G. Maluf et al., "Impact of the donor risk index on the outcome of hepatitis C virus-positive liver transplant recipients," Liver Transplant. 15(6), 592-599 (2009).

19. F. Zamboni et al., "Effect of macrovescicular steatosis and other donor and recipient characteristics on the outcome of liver transplantation," Clin. Transplant. 15(1), 53-57 (2001).

20. R. G. Knodell et al., "Formulation and application of a numerical scoring system for assessing histological activity in asymptomatic chronic active hepatitis," Hepatology 1(5), 431-435 (1981).

21. C. Melin et al., "Approach to intraoperative consultation for donor liver biopsies," Arch. Pathol. Lab Med. 137(2), 270-274 (2013).

22. V. Wadhera et al., "The impact of donor liver allograft fibrosis on patients undergoing liver transplantation," Clin. Transplant. 32(3), e13187 (2018).

23. S. Abraham and E. E. Furth, "Quantitative evaluation of histological features in" time-zero" liver allograft biopsies as predictors of rejection or graft failure: receiver-operating characteristic analysis application," Hum. Pathol. 27(10), 1077-1084 (1996).

24. Z. Hassoun et al., "Centrilobular necrosis after orthotopic liver transplantation: association with acute cellular rejection and impact on outcome," Liver Transplant. 10(4), 480-487 (2004).

25. M. K. Angele et al., "Effect of graft steatosis on liver function and organ survival after liver transplantation," Am. J. Surg. 195(2), 214-220 (2008).

26. R. Adam et al., "Deleterious effect of extended cold ischemia time on the posttransplant outcome of aged livers," Transplant. Proc. 27(1), 1181-1183 (1995).

27. D. A. Neil and S. G. Hubscher, "Current views on rejection pathology in liver transplantation," Transplant. Int. 23(10), 971-983 (2010).

28. D. A. Neil and S. G. Hubscher, "Histologic and biochemical changes during the evolution of chronic rejection of liver allografts," Hepatology 35(3), 639-651 (2002).

29. E. Rubin et al., "Effect of experimental portacaval shunt on hepatic drug metabolizing enzymes," Proc. Soc. Exp. Biol. Med. 127(2), 444-447 (1968).

30. A. Tardu et al., "Histopathological examination of explanted liver after transplantation in patients with cryptogenic cirrhosis," Transplant. Proc. 47(5), 1450-1452 (2015).

31. V. L. Venkat et al., "Recurrence of primary sclerosing cholangitis in pediatric liver transplant recipients," Liver Transplant. 20(6), 679686 (2014).

32. R. S. Young et al., "Pre-liver transplant biopsy in hepatocellular carcinoma: a potential criterion for exclusion from transplantation?" HPB (Oxford) 15(6), 418-427 (2013).

33. S. Tamura et al., "Impact of histological grade of hepatocellular carcinoma on the outcome of liver transplantation," Arch. Surg. 136(1), 25-30, discussion 31 (2001).

34. O. Adeyi, S. E. Fischer, and M. Guindi, "Liver allograft pathology: approach to interpretation of needle biopsies with clinicopathological correlation," J. Clin. Pathol. 63(1), 47-74 (2010).

35. B. Geramizadeh et al., "Histopathology of post-transplant liver biopsies, the first report from Iran," Hepat. Mon. 13(6), e9389 (2013).

36. A. Briem-Richter et al., "Liver allograft pathology in healthy pediatric liver transplant recipients," Pediatr. Transplant. 17(6), 543-549 (2013).

37. J. M. Ali et al., "Analysis of ischemia/reperfusion injury in time-zero biopsies predicts liver allograft outcomes," Liver Transplant. 21(4), 487-499 (2015).

38. M. Fiorentino et al., "Predictive value of frozen-section analysis in the histological assessment of steatosis before liver transplantation," Liver Transplant. 15(12), 1821-1825 (2009).

39. W. Wilson, "A trichrome method for staining fat with oil red $\mathrm{O}$ in frozen sections," Bull. Int. Assoc. Med. Mus. 31, 216-220 (1950).

40. S. B. Sams and J. A. Wisell, "Discordance between intraoperative consultation by frozen section and final diagnosis," Int. J. Surg. Pathol. 25(1), 41-50 (2017).

41. H. Hatami, Z. Mohsenifar, and S. N. Alavi, "The diagnostic accuracy of frozen section compared to permanent section: a single center study in Iran," Iran J. Pathol. 10(4) 295-299 (2015). 
42. S. O. Vargas, B. A. Sampson, and F. J. Schoen, "Pathologic detection of early myocardial infarction: a critical review of the evolution and usefulness of modern techniques," Mod. Pathol. 12(6), 635-645 (1999).

43. T. Ikeda et al., "Ischemic injury in liver transplantation: difference in injury sites between warm and cold ischemia in rats," Hepatology 16(2), 454-461 (1992).

44. S. Kienlein et al., "Biliary complications in liver transplantation: impact of anastomotic technique and ischemic time on short- and long-term outcome," World J. Transplant. 5(4), 300-309 (2015).

45. A. K. Singh et al., "Postoperative imaging in liver transplantation: what radiologists should know," Radiographics 30(2), 339-351 (2010).

46. K. D. Kochanek et al., "Deaths: final data for 2014," Natl. Vital Stat. Rep. 65(4), 1-122 (2016).

47. S. L. Murphy et al., "Mortality in the United States, 2014," NCHS Data Brief 2015(229), 1-8 (2015).

48. W. A. Bower et al., "Population-based surveillance for acute liver failure," Am. J. Gastroenterol. 102(11), 2459-2463 (2007).

49. H. B. El-Serag and F. Kanwal, "Epidemiology of hepatocellular carcinoma in the United States: where are we? Where do we go?" Hepatology 60(5), 1767-1775 (2014).

50. Organ Procurement and Transplantation Network, "Health Resources and Services Administration," U.S. Department of Health and Human Services National data, https://optn.transplant.hrsa.gov/data/view-datareports/build-advanced.

51. A. D'Alessandro et al., "Liver transplantation from controlled nonheart-beating donors," Surgery 128(4), 579-588 (2000).

52. H. Yersiz et al., "Assessment of hepatic steatosis by transplant surgeon and expert pathologist: a prospective, double-blind evaluation of 201 donor livers," Liver Transplant. 19(4), 437-449 (2013).

53. L. McCormack et al., "Liver transplantation using fatty livers: always feasible?" J. Hepatol. 54(5), 1055-1062 (2011).

54. K. Ishak et al., "Histological grading and staging of chronic hepatitis," J. Hepatol. 22(6), 696-699 (1995).

55. R. Adam et al., "Effect of extended cold ischaemia with UW solution on graft function after liver transplantation," Lancet 340(8832), 1373-1376 (1992).

56. T. A. Broughan and V. Douzdjian, "Donor liver selection, the SouthEastern Organ Procurement Foundation Liver Committee," Am. Surg. 64(8), 785-790 (1998).

57. H. Haga et al., "Periportal edema and necrosis as diagnostic histological features of early humoral rejection in $\mathrm{ABO}$-incompatible liver transplantation," Liver Transplant. 10(1), 16-27 (2004).

58. J. C. Hong et al., "Liver transplantation using organ donation after cardiac death: a clinical predictive index for graft failure-free survival," Arch. Surg. 146(9), 1017-1023 (2011).

59. F. F. Jobsis, "Noninvasive, infrared monitoring of cerebral and myocardial oxygen sufficiency and circulatory parameters," Science 198(4323), 1264-1267 (1977).

60. G. Giannotti et al., "Utility of near-infrared spectroscopy in the diagnosis of lower extremity compartment syndrome," J. Trauma 48(3), 396-99, discussion 399-401 (2000).

61. W. Bank et al., "Near-infrared spectroscopy in the diagnosis of mitochondrial disorders," Biofactors 7(3), 243-245 (1998).

62. C. Hock et al., "Near infrared spectroscopy in the diagnosis of Alzheimer's disease," Ann. N. Y. Acad. Sci. 777, 22-29 (1996).

63. S. Hyttel-Sorensen et al., "A phase II randomized clinical trial on cerebral near-infrared spectroscopy plus a treatment guideline versus treatment as usual for extremely preterm infants during the first three days of life (SafeBoosC): study protocol for a randomized controlled trial," Trials 14, 120 (2013).

64. R. G. Steen, K. Kitagishi, and K. Morgan, "In vivo measurement of tumor blood oxygenation by near-infrared spectroscopy: immediate effects of pentobarbital overdose or carmustine treatment," $J$. Neurooncol. 22(3), 209-220 (1994).

65. A. D. Edwards et al., "Cerebral hemodynamic effects of treatment with modified natural surfactant investigated by near infrared spectroscopy," Pediatr. Res. 32(5), 532-536 (1992).

66. J. L. Zulueta et al., "Role of intraoperative regional oxygen saturation using near infrared spectroscopy in the prediction of low output syndrome after pediatric heart surgery," J. Card. Surg. 28(4), 446-452 (2013).
67. A. Demir et al., "Intraoperative 16-channel electroencephalography and bilateral near infrared spectroscopy monitorization in aortic surgery," Turk. J. Anaesthesiol. Reanim. 43(4), 274-278 (2015).

68. B. Ruf et al., "Intraoperative renal near-infrared spectroscopy indicates developing acute kidney injury in infants undergoing cardiac surgery with cardiopulmonary bypass: a case-control study," Crit. Care 19, 27 (2015).

69. K. M. Gist et al., "A decline in intraoperative renal near-infrared spectroscopy is associated with adverse outcomes in children following cardiac surgery," Pediatr. Crit. Care Med. 17(4), 342-349 (2016).

70. G. Nogueira-Filho et al., "On site noninvasive assessment of periimplant inflammation by optical spectroscopy," J. Periodontal Res. 46(3), 382-388 (2011).

71. J. Agustsson et al., "Non-contact assessment of COD and turbidity concentrations in water using diffuse reflectance UV-Vis spectroscopy," Environ. Sci. Process Impacts 16(8), 1897-1902 (2014).

72. D. Piao et al., "Freehand diffuse optical spectroscopy imaging for intraoperative identification of major venous and arterial vessels underlying peritoneal fat: an in vivo demonstration in a pig model," J. Biomed. Opt. 22(10), 1-4 (2017).

73. M. J. Lee et al., "Liver steatosis assessment: correlations among pathology, radiology, clinical data and automated image analysis software," Pathol. Res. Pract. 209(6), 371-379 (2013).

74. T. M. Fishbein et al., "Use of livers with microvesicular fat safely expands the donor pool," Transplantation 64(2), 248-251 (1997).

75. S. Nagai et al., "Mild hepatic macrovesicular steatosis may be a risk factor for hyperbilirubinaemia in living liver donors following right hepatectomy," Br. J. Surg. 96(4), 437-444 (2009).

76. Y. Soejima et al., "Use of steatotic graft in living-donor liver transplantation," Transplantation 76(2), 344-348 (2003).

77. J. R. van Werven et al., "Assessment of hepatic steatosis in patients undergoing liver resection: comparison of US, CT, T1-weighted dual-echo MR imaging, and point-resolved 1H MR spectroscopy," Radiology 256(1), 159-168 (2010).

78. S. Zelber-Sagi et al., "Comparison of fatty liver index with noninvasive methods for steatosis detection and quantification," World $J$. Gastroenterol. 19(1), 57-64 (2013).

79. A. Chauhan et al., "Diagnostic accuracy of hepatorenal index in the detection and grading of hepatic steatosis," J. Clin. Ultrasound 44(9), 580-586 (2016).

80. D. J. Evers et al., "Diffuse reflectance spectroscopy: toward real-time quantification of steatosis in liver," Transpl. Int. 28(4), 465-474 (2015).

81. A. C. Westerkamp et al., "Diffuse reflectance spectroscopy accurately quantifies various degrees of liver steatosis in murine models of fatty liver disease," J. Transl. Med. 13, 309 (2015).

82. J. H. Nilsson et al., "Diffuse Reflectance Spectroscopy for Surface Measurement of Liver Pathology," Eur. Surg. Res. 58(1-2), 40-50 (2017).

83. T. Kitai, B. Beauvoit, and B. Chance, "Optical determination of fatty change of the graft liver with near-infrared time-resolved spectroscopy," Transplantation 62(5), 642-647 (1996).

84. B. L. McLaughlin et al., "Electrical and optical spectroscopy for quantitative screening of hepatic steatosis in donor livers," Phys. Med. Biol. 55(22), 6867-6879 (2010).

85. D. Piao et al., "In vivo assessment of diet-induced rat hepatic steatosis development by percutaneous single-fiber spectroscopy detects scattering spectral changes due to fatty infiltration," J. Biomed. Opt. 20(11), 117002 (2015).

86. D. Piao, "In vivo percutaneous reflectance spectroscopy of fatty liver development in rats suggests that the elevation of the scattering power is an early indicator of hepatic steatosis," J. Innovative Opt. Health Sci. 11(4), 1850019 (2018).

87. D. S. Stewart, "Investigation of the optical properties of the lenses spontaneously regenerated after removal of the contents of the lens capsule of the eyes of adult rabbits," Trans. Ophthalmol. Soc. U. K. 85, 655-660 (1965).

88. M. L. Onozato et al., "Optical coherence tomography of human kidney," J. Urol. 183(5), 2090-2094 (2010).

89. D. Piao et al., "Effect of capsule on surface diffuse reflectance spectroscopy of capsular solid organs," Proc. SPIE 10468, 104680B (2018). 
90. D. Piao et al., "Effects of capsule on surface diffuse reflectance spectroscopy of the subcapsular parenchyma of a solid organ," J. Biomed. Opt. 23(12), 121602 (2018).

91. Y. He et al., "Toward surface quantification of liver fibrosis progression," J. Biomed. Opt. 15(5), 056007 (2010).

92. U. O. Goel et al., "Feasibility of quantitative diffuse reflectance spectroscopy for targeted measurement of renal ischemia during laparoscopic partial nephrectomy," J. Biomed. Opt. 19(10), 107001 (2014).

93. S. Xu et al., "Quantification of liver fibrosis via second harmonic imaging of the Glisson's capsule from liver surface," J. Biophotonics 9(4), 351-363 (2016).

94. D. A. Fabila-Bustos et al., "Diffuse reflectance spectroscopy as a possible tool to complement liver biopsy for grading hepatic fibrosis in paraffin-preserved human liver specimens," Appl. Spectrosc. 68(12), 1357-1364 (2014).

Daqing Piao is a professor in the School of Electrical and Computer Engineering (ECE) at Oklahoma State University (OSU). He received his PhD and MS degrees in biomedical engineering in 2003 and 2001, respectively, from the University of Connecticut and BS degree in physics in 1990 from Tsinghua University. He has been on the faculty of ECE at OSU since 2005. His group develops biophotonics technologies to improve the outcomes of surgery, with specific interest in pretransplant donor liver assessment.
Alan Hawxby received his medical degree from the University of Oklahoma, followed by surgery residency at the University of Missouri-Kansas City, and a transplant surgery fellowship at Johns Hopkins Hospital, Baltimore. He is currently a clinical associate professor and surgical director of adult and pediatric transplantation at the University of Oklahoma. He is a liver, kidney, and pancreas transplant surgeon.

Harlan Wright is the medical director of the liver transplant program at the Oklahoma Transplant Services of OUHSC. He received his medical degree from Universidad Central de Venezuela, completing his postgraduate gastroenterology training at the University Hospital of Caracas. He spent many years at the University of Pittsburgh Transplant Center and is published in many scientific journals. He treats patients with liver disease and patients who have undergone liver transplants.

Erin M. Rubin holds the James Park Dewar, MD, professorship of pathology in the Department of Pathology at OUHSC. She is a GI/hepatopancreaticobiliary and transplant pathologist. Her daily clinical responsibilities involve the diagnostic interpretation and analysis of gross and microscopic pathology material. She serves on the admission committee at the medical school of OUHSC. She is also a course director and associate course director for medical students within the department. 